\title{
Asymptotic Behavior of Pseudo-Resolvents on Some Grothendieck Spaces
}

By

\author{
Sen-Yen SHAW*
}

\begin{abstract}
Albstract
For a pseudo-resolvent $\left\{J_{\lambda} ; \lambda \in \Omega \subset C\right\}$ of operators on a Grothendieck space $X$, it is proved that the strong convergence of $\lambda J_{\lambda}$ as $\lambda \rightarrow 0$ [resp. $\left.|\lambda| \rightarrow \infty\right]$ is equivalent to that $\| \lambda J_{\lambda}||=\mathrm{O}(1)(\lambda \rightarrow 0)[$ resp. $|\lambda| \rightarrow \infty]$ and $\overline{R\left(\lambda J_{\lambda}^{*}-I^{*}\right)}=\mathrm{w}^{*}-c l\left(R\left(\lambda J_{\lambda}^{*}-I^{*}\right)\right)\left[\right.$ resp. $\overline{R\left(J_{\lambda}^{*}\right)}=$ $\left.\mathrm{w}^{*}-\operatorname{cl}\left(R\left(J_{\lambda}^{*}\right)\right)\right]$. If, in addition, $X$ has the Dunford-Pettis property, then the strong convergence implies the uniform convergence. It is also shown that if a semigroup of class $(E)$ on such a space is strongly Abel-ergodic at zero, then it must be uniformly continuous.
\end{abstract}

\section{$\S 1$. Introduction}

Let $\mathscr{B}(X)$ denote the set of all bounded linear operators on a Banach space $X$. A family $\left\{J_{\lambda} ; \lambda \in \Omega\right\}$ of operators in $\mathscr{B}(X)$ is called a pseudo-resolvent on $\Omega \subset C$ if

$$
J_{\lambda}-J_{\mu}=(\mu-\lambda) J_{\lambda} J_{\mu}, \quad(\lambda, \mu \in \Omega) .
$$

It is known that the ranges $R\left(J_{\lambda}\right), R\left(\lambda J_{\lambda}-I\right)$, and the null spaces $N\left(J_{\lambda}\right), N\left(\lambda J_{\lambda}-I\right)$ are independent of the parameter $\lambda$ (cf. $[6$, p. 215]).

The strong convergence and the uniform convergence of $\lambda J_{\lambda}$ as $\lambda \rightarrow 0$ or $|\lambda| \rightarrow \infty$ have been studied in Yosida [7] and Shaw [5], respectively. The results were obtained for general Banach spaces. In this note we investigate the strong convergence of $\lambda J_{\lambda}$ on a Grothendieck space and the uniform convergence on a Grothendieck space with the Dunford-Pettis property.

A Banach space $X$ is called a Grothendieck space if every $\mathrm{w}^{*}$-convergent sequence in the dual space $X^{*}$ is weakly convergent. $X$ is said to have the Dunford-Pettis property if $\left\langle x_{n}, x_{n}^{*}\right\rangle \rightarrow 0$ whenever $\left\{x_{n}\right\} \subset X$ tends weakly to 0 and $\left\{x_{n}^{*}\right\} \subset X^{*}$ tends weakly to 0 . Examples of a Grothendieck space with the Dunford-Pettis property include $L^{\infty}, B(S, \Sigma), H^{\infty}(D)$, etc. (see [3].) On such a space, the weak convergence, the strong convergence, and the uniform con-

Communicated by S. Matsuura, August 14, 1987.

* Department of Mathematics, National Central University, Chung-Li, Taiwan, Republic of China. 
vergence of $\lambda J_{\lambda}$ are seen to be equivalent. This is similar to the recent results of Lotz [2,3] and of Shaw [4] on the continuity and the ergodicity of operator semigroups and of cosine operator functions, repectively.

\section{§ 2. Strong Ergodic Theorems}

We shall denote by $P$ [resp. $Q$ ] the mapping: $x \rightarrow \mathrm{s}-\lim _{\lambda \rightarrow 0} \lambda J_{\lambda} x$ [resp. s-lim| $\left.\lim _{|\lambda| \rightarrow \infty} \lambda J_{\lambda} x\right]$. First, for the sake of convenience we state Yosida's theorem [7, pp. 217-218] in the following form:

Theorem 1. If $\left\|\lambda J_{\lambda}\right\|=O(1)(\lambda \rightarrow 0)[$ resp. $|\lambda| \rightarrow \infty]$ and $0 \in \bar{\Omega}[$ resp. $\Omega$ is unbounded], then $P$ [resp. $Q]$ is a bounded linear projection with $R(P)=N\left(\lambda J_{\lambda}-I\right)$ and $N(P)=\overline{R\left(\lambda J_{\lambda}-I\right)}\left[\right.$ resp. $R(Q)=\overline{R\left(J_{\lambda}\right)}$ and $\left.N(Q)=N\left(J_{\lambda}\right)\right]$. Moreover, $x \in D(P)$ $[$ resp. $D(Q)]$ if and only if there is a sequence $\lambda_{n}$ tending to $0[$ resp. $\infty]$ such that $\mathrm{w}-\lim _{n \rightarrow \infty} \lambda_{n} J_{\lambda_{n}} x$ exists.

It follows from the last assertion that strong convergence and weak convergence are equivalent. Also, $\lambda J_{\lambda}$ is strongly convergent whenever $X$ is a reflexive space. Since $D(P)^{\perp}=\left\{N\left(\lambda J_{\lambda}-I\right) \oplus \overline{R\left(\lambda J_{\lambda}-I\right)}\right\}^{\perp}=N\left(\lambda J_{\lambda}-I\right)^{\perp} \cap$ $N\left(\lambda J_{\lambda}^{*}-I^{*}\right), \lambda J_{\lambda}$ is strongly convergent as $\lambda \rightarrow 0$ if and only if $\left\|\lambda J_{\lambda}\right\|=O(1)(\lambda \rightarrow 0)$, and $N\left(\lambda J_{\lambda}-I\right)$ separates $N\left(\lambda J_{\lambda}^{*}-I^{*}\right)$ (i.e. $N\left(\lambda J_{\lambda}-I\right)^{\perp} \cap N\left(\lambda J_{\lambda}^{*}-I^{*}\right)=\{0\}$ ).

The following theorem gives another characterization of the strong convergence of $\lambda J_{\lambda}$ in the case that $X$ is a Grothendieck space.

Theorem 2. Let $X$ be a Grothendieck space, $\lambda J_{\lambda}$ is convergent in the strong operator topology as $\lambda \rightarrow 0[$ resp. $|\lambda| \rightarrow \infty]$ if and only if $\left\|\lambda J_{\lambda}\right\|=O(1)$ $(\lambda \rightarrow 0)[$ resp. $|\lambda| \rightarrow \infty]$ and $\left.\overline{R\left(\lambda J_{\lambda}^{*}-I^{*}\right.}\right)=w^{*}-\operatorname{cl}\left(R\left(\lambda J_{\lambda}^{*}-I^{*}\right)\right)\left[\operatorname{resp} . \overline{R\left(J_{\lambda}^{*}\right)}=\right.$ $\left.w^{*}-\operatorname{cl}\left(R\left(J_{\lambda}^{*}\right)\right)\right]$.

Proof. We only prove the case " $\lambda \rightarrow 0$;" a similar argument works for the other case " $|\lambda| \rightarrow \infty$."

First, suppose that $P=$ so- $\lim _{\lambda \rightarrow 0} \lambda J_{\lambda}$ exists. Then clearly one has $\left\|\lambda J_{\lambda}\right\|=O(1)$ $(\lambda \rightarrow 0)$, by the uniform boundedness principle. $X$ being Grothendieck, it follows that $\mathrm{w}-\lim _{n \rightarrow \infty} \lambda_{n} J_{\lambda_{n}}^{*} x^{*}=\mathrm{w}^{*}-\lim _{n \rightarrow \infty} \lambda_{n} J_{\lambda_{n}}^{*} x^{*}=P^{*} x^{*}$ for any sequence $\left\{\lambda_{n}\right\} \rightarrow 0$ and any $x^{*} \in X^{*}$. Applying Theorem 1 to the pseudo-resolvent $\left\{J_{\lambda}^{*}\right\}$ we see that $P^{*}=$ so- $\lim _{\lambda \rightarrow 0} \lambda J_{\lambda}^{*}$. Hence we have

$$
\begin{aligned}
\overline{R\left(\lambda J_{\lambda}^{*}-I^{*}\right)} & =N\left(P^{*}\right)=R(P)^{\perp}=N\left(\lambda J_{\lambda}-I\right)^{\perp} \\
& =\left[{ }^{\perp} R\left(\lambda J_{\lambda}^{*}-I^{*}\right)\right]^{\perp}=w^{*}-c l\left(R\left(\lambda J_{\lambda}^{*}-I^{*}\right)\right) .
\end{aligned}
$$


Conversely, if $\left\|\lambda J_{\lambda}\right\|=O(1)(\lambda \rightarrow 0)$ and $\overline{R\left(\lambda J_{\lambda}^{*}-I^{*}\right)}=w^{*}-\operatorname{cl}\left(R\left(\lambda J_{\lambda}^{*}-I^{*}\right)\right.$ $\left(=N\left(\lambda J_{\lambda}-I\right)^{\perp}\right)$, then Theorem 1, applied to $\left\{J_{\lambda}\right\}$ and $\left\{J_{\lambda}^{*}\right\}$, implies that $D(P)=N\left(\lambda J_{\lambda}-I\right) \oplus \overline{R\left(\lambda J_{\lambda}-I\right)}$ and $\overline{R\left(\lambda J_{\lambda}^{*}-I^{*}\right)} \cap N\left(\lambda J_{\lambda}^{*}-I^{*}\right)=\{0\}$, so that $D(P)^{\perp}=\{0\}$. This shows that $D(P)=X$ because it is closed.

\section{§3. Uniform Ergodic Theorems}

For a pseudo-resolvent $\left\{J_{\lambda}\right\}$ on a general Banach space $X$, the uniform convergence of $\lambda J_{\lambda}$ is characterized in the following theorem, which was proved in [5].

Theorem 3. (i) uo- $\lim _{\lambda \rightarrow 0} \lambda J_{\lambda}$ exists if and only if $\left\|\lambda^{2} J_{\lambda}\right\| \rightarrow 0$ as $\lambda \rightarrow 0$ and $R\left(\lambda J_{\lambda}-I\right)$ is closed.

(ii) uo- $\lim _{|\lambda| \rightarrow \infty} \lambda J_{\lambda}=Q$ exists if and only if $\left\|J_{\lambda}\right\| \rightarrow 0$ as $|\lambda| \rightarrow \infty$ and $R\left(J_{\lambda}\right)$ is closed, if and only if $J_{\lambda}=Q(\lambda I-A)^{-1}$ where $Q^{2}=Q, A \in B(X)$ and $A Q=Q A=A$.

In general the strong convergence of $\lambda J_{\lambda}$ is weaker than the uniform convergence. But it is to be shown that these two kinds of convergence coincide in the class of Grothendieck spaces with the Dunford-Pettis property. To prove this we need the following lemma of Lotz [3].

Lemma 4. Let $\left\{V_{n}\right\}$ be a sequence of operators on a Banach space $X$ with the Dunford-Pettis property. Suppose that w-lim $V_{n} x_{n}=0$ for every bounded sequence $\left\{x_{n}\right\}$ in $X$ and $\mathrm{w}-\lim V_{n}^{*} x_{n}^{*}=0$ for every bounded sequence $\left\{x_{n}^{*}\right\}$ in $X^{*}$. Then $\left\|V_{n}^{2}\right\| \rightarrow 0$. In particular, $V_{n}-I$ and $V_{n}+\mathbb{I}$ are invertible for large $n$.

Theorem 5. Let $\left\{J_{\lambda}\right\}$ be a pseudo-resolvent on a Grothendieck space $X$ with the Dunford-Pettis property. The following statements are equivalent:

(1) $\left\|\lambda J_{\lambda}\right\|=O(1)(\lambda \rightarrow 0)$ and for each $x \in X$ there is a sequence $\lambda_{n} \rightarrow 0$ such that $\mathrm{w}-\lim _{n \rightarrow \infty} \lambda_{n} J_{\lambda_{n}} x$ exists.

(2) $P:=$ so- $\lim _{\lambda \rightarrow 0} \lambda J_{\lambda}$ exists.

(3) $\left\|\lambda J_{\lambda}-P\right\| \rightarrow 0$ as $\lambda \rightarrow 0$.

(4) $\left\|\lambda^{2} J_{\lambda}\right\| \rightarrow 0$ as $\lambda \rightarrow 0$, and $\overline{R\left(\lambda J_{\lambda}-I\right)}$ is closed.

(5) $\left\|\lambda J_{\lambda}\right\|=O(1)(\lambda \rightarrow 0)$ and $R\left(\lambda J_{\lambda}^{*}-I^{*}\right)=w^{*}-\operatorname{cl}\left(R\left(\lambda J_{\lambda}^{*}-I^{*}\right)\right)$.

Proof. " $(1) \Leftrightarrow(2) "$, " (2) $\Leftrightarrow(5) "$, and "(3) $\Leftrightarrow(4)$ " are contained in Theorem 1, Theorem 2, and Theorem 3 (i), respectively. Thus it remains to show that (2) implies (3).

Suppose (2) holds. Then Theorem 1 implies that $X=R(P) \oplus N(P)$ and 
$R(P)=N\left(\lambda J_{\lambda}-I\right)$ for all $\lambda \in \Omega$. So, in order to prove that $\left\|\lambda J_{\lambda}-P\right\| \rightarrow 0$, it is no loss of generality to assume that $P=0$.

Let $V_{n}=n^{-1} J_{1 / n}$. Then s-lim $V_{n} x=P x=0$ for all $x \in X$ so that $\left\{V_{n}^{*} x_{n}^{*}\right\}$ converges weakly* and hence weakly to zero for any bounded sequence $\left\{x_{n}^{*}\right\}$ in $X^{*}$. In particular, $\left\{n^{-1} J_{1 / n}^{*} x^{*}\right\}$ converges weakly to zero for all $x^{*} \in X^{*}$. Now Theorem 1 applies to $\left\{J_{\lambda}^{*}\right\}$ to yield that $\left\{V_{n}^{*} x^{*}\right\}$ converges strongly to zero for all $x^{*} \in X^{*}$. Hence $\left\{V_{n} x_{n}\right\}$ converges weakly to zero for any bounded sequence $\left\{x_{n}\right\}$ in $X$. It follows from Lemma 4 that $V_{n}-I$ is invertible for large $n$.

Finally, it follows from the estimate

$$
\begin{aligned}
\left\|\lambda J_{\lambda}\right\| & \leqslant\left\|\lambda J_{\lambda}\left(n^{-1} J_{1 / n}-I\right)\right\|\left\|\left(V_{n}-I\right)^{-1}\right\| \\
& =\left\|\left(\frac{1}{n}-\lambda\right)^{-1}\left[\lambda^{2} J_{\lambda}-\frac{\lambda}{n} J_{1 / n}\right]\right\|\left\|\left(V_{n}-I\right)^{-1}\right\|
\end{aligned}
$$

that $\left\|\lambda J_{\lambda}\right\| \rightarrow 0$ as $\lambda \rightarrow 0$. This proves the theorem.

If we let $V_{n}$ be $n J_{n}-I$, then a similar argument as above, together with Theorems 1, 2, and 3 (ii), will give the following uniform ergodic theorem for the case " $|\lambda| \rightarrow \infty$ ". This is a slight extension of a result of Lotz [2] which treated the case $Q=I$ and did not include conditions (4) and (5).

Theorem 6. Let $X$ be a Grothendieck space with the Dunford-Pettis property. The following statements are equivalent:

(1) $\left\|\lambda J_{\lambda}\right\|=O(1)(|\lambda| \rightarrow \infty)$, and for each $x \in X$ there is a sequence $\left\{\lambda_{n}\right\}$, $\left|\lambda_{n}\right| \rightarrow \infty$, such that $\mathrm{w}-\lim _{n \rightarrow \infty} \lambda_{n} J_{\lambda_{n}} x$ exists.

(2) $Q:=s o-\lim _{|\lambda| \rightarrow \infty} \lambda J_{\lambda}$ exists.

(3) $\left\|\lambda J_{\lambda}-Q\right\| \rightarrow 0$ as $|\lambda| \rightarrow \infty$.

(4) $\left\|J_{\lambda}\right\| \rightarrow 0$ as $|\lambda| \rightarrow \infty$, and $R\left(J_{\lambda}\right)$ is closed.

(5) $\quad\left\|\lambda J_{\lambda}\right\|=O(1)(|\lambda| \rightarrow \infty)$ and $\overline{R\left(J_{\lambda}^{*}\right)}=w^{*}-\operatorname{cl}\left(R\left(J_{\lambda}^{*}\right)\right)$.

(6) $J_{\lambda}=Q(\lambda I-A)^{-1}$ for some $Q, A \in \mathscr{B}(X)$ satisfying $Q^{2}=Q, A Q=Q A=A$.

\section{§ 4. Uniform Continuity of Semigroups and Cosine Functions}

Lotz [2] has proved that every semigroup of class $(A)$ in the sense of $[1, \mathrm{p}$. 342] on a Grothendieck space with the Dunford-Pettis property is uniformly continuous. In what follows we shall apply Theorem 6 and a theorem of Hille [1, Theorem 18.8.3] to deduce a slight generalization.

A semigroup $\{T(t) ; t>0\}$ of type $w_{0}$ is said to be of class $(E)$ if

(a) $T(\cdot)$ is strongly continuous on $(0, \infty)$; 
(b) $X_{0}:=\left\{x ; \int_{0}^{1}\|T(t) x\| d t<\infty\right\}$ is dense in $X$;

(c) the linear operator $R(\lambda) x:=\int_{0}^{\infty} e^{-\lambda t} T(t) x d t$ is defined on $X_{0}$ for each $\lambda>w_{0}$, (see [1, p. 509]). It is known that $\left\{R(\lambda) ; \lambda>w_{0}\right\}$ is a pseudo-resolvent (cf. [1, p. 510]).

$T(\cdot)$ is said to be strongly (resp. uniformly) Abel-ergodic to $Q$ at zero if $\lambda R(\lambda)$ converges to $Q$ in the strong (resp. uniform) operator topology as $\lambda$ tends to infinity. Theorem 18.8 .3 of [1] asserts that if $T(\cdot)$ is of class $(E)$ and is uniformly Abel-ergodic to $Q$ at zero, then $T(t)=Q \exp (t A)$ with $Q^{2}=Q, A \in \mathscr{B}(X)$ and $A Q=Q A=A$. We combine this with Theorem 6 to formulate the following result.

Corollary 7. Let $T(\cdot)$ be a semigroup of class $(E)$ on a Grothendieck space with the Dunford-Pettis property. If $T(\cdot)$ is strongly Abel-ergodic $10 Q$ at zero, then $T(t)=Q \exp (t A)$ where $Q^{2}=Q, A \in \mathcal{B}(X)$ and $A Q=Q A=A$.

In particular, if $T\left({ }^{\circ}\right)$ is a semigroup of class $(A)$, then $T\left({ }^{\circ}\right)$ belongs to the class $(E)$ and it is strongly Abel-ergodic to $I$ at zero. Corollary 7 shows that $T(t)=\exp (t A)$ with $A \in \mathscr{B}(X)$ and so is uniformly continuous.

We close this section with another application of Theorems $1 \& 6$. Let $A$ be the generator of a strongly continuous cosine operator function $C\left({ }^{\circ}\right)$ on a Grothendieck space with the Dunford-Pettis property. Then $\overline{D(A)}=X$, and there are constants $M>0$ and $w>0$ such that $\lambda^{2} \in \rho(A)$ and $\left\|\lambda\left(\lambda^{2}-A\right)^{-1}\right\| \leq$ $M /(\lambda-w)$ for all $\lambda>w$. With $J_{\lambda}:=(\lambda-A)^{-1}$, Theorems $1 \& 6$ show that $A$ is bounded and hence $C(\cdot)$ is uniformly continuous (cf. [6]). This proves the following corollary which has appeared in [4] with a different proof.

Corollary 8. Every strongly continuous cosine operator function on a Grothendieck space with the Dunford-Pettis property is uniformly continuous.

\section{References}

[1] Hille, E. and Phillips, R.S., Functional Analysis and Semi-groups, Amer. Math. Soc. Colloq. Publ., vol. 31, Amer. Math. Soc., Providence, R.I., 1957.

[2] Lotz, H.P., Uniform convergence of operators on $L^{\infty}$ and similar spaces, Math. Z., 190 (1985), 207-220.

[3] Tauberian theorems for operators on $L^{\infty}$ and similar spaces, Functional Analysis: Surveys and Recent Results, $\mathbb{1 1 1}$ (1984), 117-133.

[4] Shaw, S.-Y., On $w^{*}$-continuous cosine operator functions, J. Funct. Anal. 66 (1986), 73-95.

[5] - Uniform ergodic theorems for locally integrable semigroups and pseudoresolvents, Proc. Amer. Math. Soc., 98 (1986), 61-67. 
[6] Travis, C.C. and Webb, G.F., Compactness, regularity, and uniform continuity properties of strongly continuous cosine families, Houston J. Math., 3 (1977), 555-567.

[7] Yosida, K., Functional Analysis, 3rd ed., Springer-Verlag, New York, 1971. 